\title{
Perioperative anaphylaxis to intravenous paracetamol caused by allergy to mannitol - a hidden allergen
}

\author{
Melchiors BLB, Krøigaard M, Garvey LH
}

Danish Anaesthesia Allergy Centre (DAAC), Allergy Clinic, Department of Dermatology and Allergy, Herlev and Gentofte Hospital, University of Copenhagen, Denmark

Background

Allergy to excipients is rare but often overlooked due to lack of awareness of excipients and their allergenic potential.

Excipients have many different properties and act as eg preservatives, stabilizers, sweeteners or to increase solubility.

In this case we present a patient who developed perioperative anaphylaxis (PA) shortly after administration of iv paracetamol (PCM). It highlights the importance of specialized investigation and of testing with the exact formulation of drugs.

\section{Case}

A 27 year old woman was referred for investigation in the Danish Anaesthesia Allergy Centre (DAAC) due to anaphylaxis after a minor surgical procedure. She had a history of chronic urticaria with daily symptoms, and exacerbations 1-2 times/week. She had experienced worsening urticaria when taking the antihistamine desloratadine (Aerius $\left.{ }^{\circledR}\right)$.

Induction and anaesthesia with propofol, remifentanil and fentanyl were
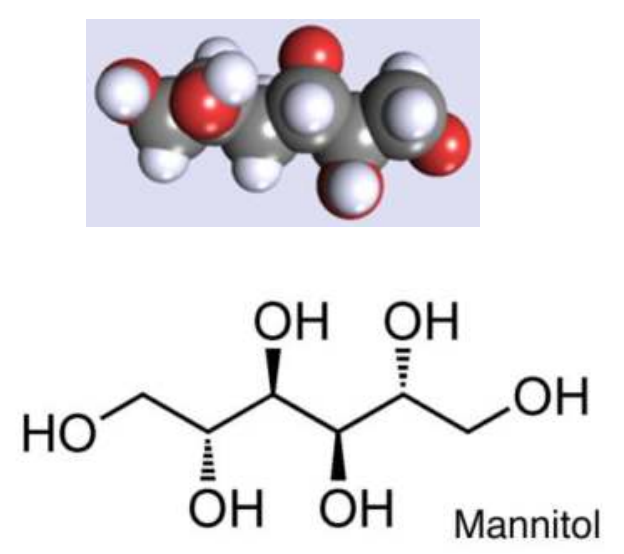
uneventful. During recovery, just after removal of the laryngeal mask and 5 minutes after administration of iv PCM, she developed severe hypotension (55/20), tachycardia (175), universal flushing and pruritus.

Anaphylaxis was suspected and treatment was started.

A total dose of $160 \mathrm{mcg}$ iv adrenalin, clemastin $2 \mathrm{mg}$, methylprednisolone $80 \mathrm{mg}$ and 2 I fluids were given. She required adrenalin infusion for 14 hours in ICU, and developed both angioedema and peripheral edema, but no respiratory symptoms. Serum tryptase 1 hour and 20 minutes after the reaction was elevated at $11.9 \mathrm{mcg} / \mathrm{l}$, baseline level $6.51 \mathrm{mcg} / \mathrm{l}$. She was discharged next day from ICU.

Subsequent investigations in DAAC revealed positive skin testing for iv PCM and the excipient mannitol. Oral provocation with tablet PCM was negative. She was advised to avoid foods and drugs containing mannitol eg diet coke, mushrooms, cauliflower and desloratadine and her exacerbations of urticaria were reduced. All other perioperative exposures tested negative.

\section{Discussion}

In this case allergy to mannitol, an excipient in iv PCM, was diagnosed. The allergy would have been missed if testing with the iv preparation of PCM had not been performed. This highlights the importance of retrieving detailed information about all exposures prior to PA. PA should always be investigated systematically in specialist centres with collaboration between allergists and anaesthesiologists.

\section{Learning points}

Anaphylaxis in the perioperative setting is complex and subsequent investigations are required with focus on all possible causes including

hidden allergens 